\title{
An unusual mutation in RECQ4 gene leading to Rothmund-Thomson syndrome
}

\author{
Pauline Balraj ${ }^{\mathrm{a}}$, Pat Concannon ${ }^{\mathrm{b}}$, Rahman Jamal ${ }^{\mathrm{c}}$, Alessandro Beghini ${ }^{\mathrm{d}}$, \\ T.S. Hoe ${ }^{\mathrm{e}}$, Alan Soobeng Khoo ${ }^{\text {a }}$, Ludovica Volpi ${ }^{\mathrm{a}, \mathrm{d}, *}$ \\ a Division of Molecular Pathology, Cancer Research Centre, Institute for Medical Research, 50588 Kuala Lumpur, Malaysia \\ ${ }^{\mathrm{b}}$ Molecular Genetics Program, Virginia Mason Research Centre, 1201 Ninth Avenue, Seattle, WA 98101-2795, USA \\ ${ }^{\mathrm{c}}$ Department of Paediatrics, Faculty of Medicine, National University of Malaysia, Kuala Lumpur, Malaysia \\ ${ }^{\mathrm{d}}$ Department of Biology and Genetics for Medical Sciences, University of Milan, Via Viotti 5, 20133 Milan, Italy \\ e Paediatrics Clinic, Selangor Medical Centre, Selangor, Malaysia
}

Received 15 May 2002; received in revised form 29 July 2002; accepted 7 August 2002

\begin{abstract}
Rothmund-Thomson syndrome (OMIM \#268400) is a severe autosomal recessive genodermatosis: characterised by growth retardation, hyperpigmentation and frequently accompanied by congenital bone defects, brittle hair and hypogonadism. Mutations in helicase RECQ4 gene are responsible for a subset of cases of RTS. Only six mutations have been reported, thus, far and each affecting the coding sequence or the splice junctions. We report the first homozygous mutation in RECQ4 helicase: 2746-2756-delTGGGCTGAGGC in IVS8 responsible for the severe phenotype associated with RTS in a Malaysian pedigree. We report also a $5321 \mathrm{G} \rightarrow$ A transition in exon 17 and the updated list of the RECQ4 gene mutations.
\end{abstract}

(C) 2002 Elsevier Science B.V. All rights reserved.

Keywords: Rothmund-Thomson syndrome; RECQ4; Intronic mutation; Splicing

\section{Introduction}

Rothmund-Thomson syndrome (OMIM \#268400) shows many analogies with Werner (OMIM \# 277700) and Bloom (OMIM \#210900) syndromes. These autosomal recessive disorders are associated with genomic instability and a predisposition to malignancy and/or premature ageing. WRN gene is defective in Werner syndrome, BLM in Bloom syndrome and RECQ4 has been associated to Rothmund-Thomson syndrome. Many evidences link Rothmund-Thomson syndrome

\footnotetext{
* Corresponding author. Tel.: +39-2-503-15849; fax: +39-2-503-15864.

E-mail address: ludovica.volpi@unimi.it (L. Volpi).
}

to helicases RECQ4: three individuals from two pedigrees [1] presented the first mutations in RECQ4 gene, and recently another family with two affected individuals. All these patients carried two compound mutations in the gene [2]. Moreover, some RTS patients had no mutations in the RECQ4 helicase. These data suggest a certain degree of heterogeneity in the syndrome [3]. Contract grant sponsor: Top Down project under the Ministry of Science, Technology and Environment, Malaysia; Contract Grant number: 06-05-01-T001, AIRC.

The inbred Malaysian pedigree (Fig. 1) that we present in this paper shows an unusual mutation in RECQ4 gene in two fraternal twins in the fourth generation, while both the parents and the grand- 


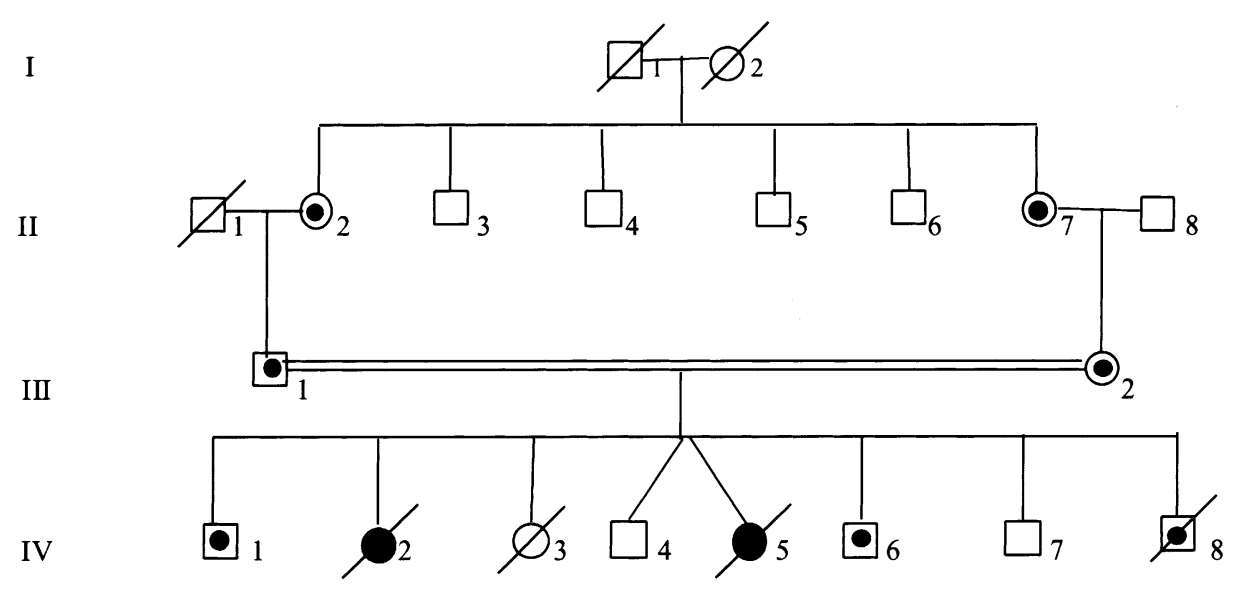

Fig. 1. Pedigree of the Malaysian family. In the second generation, the two sisters II-2 and II-7 probably inherited the 2746-2756-delTGGGCTGAGGC deletion and the $5321 \mathrm{G} \rightarrow$ A transition from one parent. In the third generation, III-1 and III-2 inherited the allele carrying the two mutations. In the fourth generation, individuals IV-1, IV-6 and IV-8 inherited one copy of the same allele. IV-4 and IV-5 inherited two copies of the deleted allele. Individuals I-1, I-2, II-1, IV-2 and IV-3 died before the genetic testing, and individuals II-3, II-4, II-5, II-6 were not tested. IV-2 died at age of 7 years due to osteosarcoma, IV-3 died due to pneumonia, IV-8 died at 4 months. Closed symbols denote clinically affected individuals and dotted symbols the carriers.

(A)
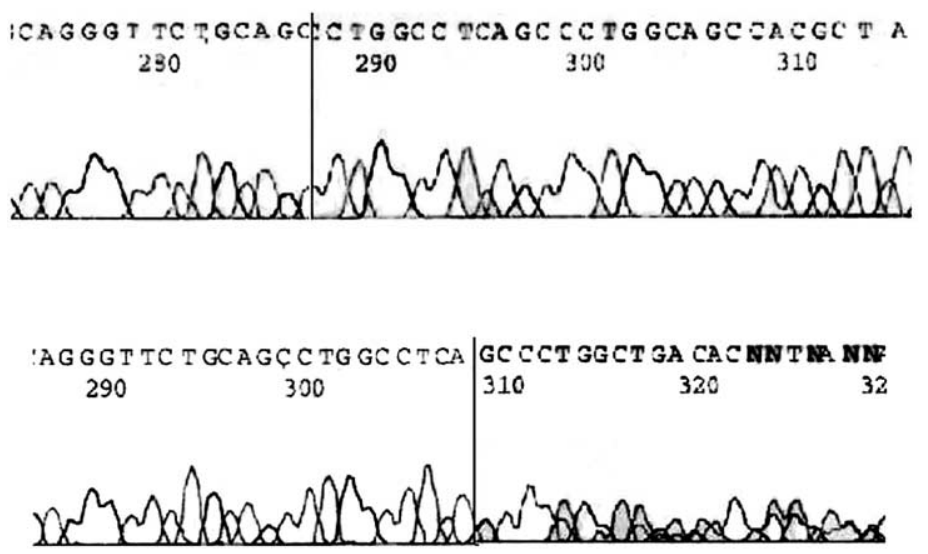

(B)

GAGC CT GC CGCA 200

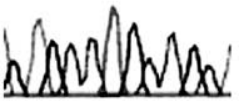

Fig. 2. (A) The homozygous (top) 2746-2756-delTGGGCTGAGGC mutation detected in individuals IV-4 and IV-5; (bottom) the same mutation in heterozygosity in III-1, III-2, IV-1, IV-6 and IV-8. (B) The homozygous (top) $5321 \mathrm{G} \rightarrow$ A transition in individual IV-4 and IV-5; (bottom) the same mutation in heterozygosity in III-1, III-2, IV-1, IV-6 and IV-8.

mothers are heterozygous carriers for the mutation: a 2746-2756-delTGGGCTGAGGC (Fig. 2A) in IVS8. We also describe a $5321 \mathrm{G} \rightarrow$ A transition leading to R1006Q missense mutation present in the same family. The RECQ4 allele containing the deletion does not produce a normal transcript, and the sequence of the RT-PCR products shows that IVS8 is not spliced correctly in the deleted alleles. This is the first homozygous mutation reported in RECQ4 gene associated with the Rothmund-Thomson syndrome. 
Table 1

\begin{tabular}{|c|c|c|c|}
\hline$T_{\mathrm{m}}\left({ }^{\circ} \mathrm{C}\right)$ & bp & Forward primer & Reverse primer \\
\hline 61 & 465 & g34FP 5'-GGAGATTCGCTGGACGAT-3' & g499RP 5'-AAACAGGGAAGTGGGAGGAG-3' \\
\hline 55 & 440 & FP121 5'-GCACTCTGAAGCGTACCACG-3' & RP334 5'-CTGCAGGGTGCCTTTCAGATT-3' \\
\hline \multirow[t]{2}{*}{62} & 523 & 1F 5'-AATCGGGCTGCGACCAAGAG-3' & 1R 5'-AGCTGTGGAGGCTCATCACT-3' \\
\hline & & \multicolumn{2}{|c|}{ Internal primer in primer 1F/1R product: FP241 5'-ACCAAGAGTCCACAGCCTAC-3' } \\
\hline 56 & 198 & FP392 5'-GAAGAGCCTCATCTAAGGCA-3' & RP571 5'-TCACTGTGACATCGCTGTAA-3' \\
\hline 62 & 867 & 2F 5'-AGTGATGAGCCTCCACAGCT-3' & 4R 5'-CCAGTGATCGAACTGCTCGTT-3' \\
\hline 58 & 274 & 2F 5'-AGTGATGAGCCTCCACAGCT-3' & 2R 5'-ATGCTGACTTCTTGGAAG-3' \\
\hline 62 & 524 & 2F 5'-AGTGATGAGCCTCCACAGCT-3' & 3R 5'-AGCCCTAGCTTGACTGGAGG-3' \\
\hline 62 & 610 & 3F 5'-CTTCCAAGAAGTCAGCATCC-3' & 4R 5'-CCAGTGATCGAACTGCTCGTT-3' \\
\hline 62 & 362 & 4F 5'-CCTCCAGTCAAGCTAGGGCT-3' & 4R 5'-CCAGTGATCGAACTGCTCGTT-3' \\
\hline 57 & 741 & FP132 5'-AGCATGGAAGCAGAAGTGGC-3' & 5R 5'-TGGAGATGCCAGACAGGATC-3' \\
\hline 55 & 657 & 5F 5'-AACGAGCAGTTCGATCACTGG-3' & 5R 5'-TGGAGATGCCAGACAGGATC-3' \\
\hline \multirow[t]{2}{*}{63} & 785 & FP1387 5'-ATCTGTGTCTGCAGAGACGCC-3 & Q4C3R 5'-TGGTCATGCCCGAGTGTATGC-3' \\
\hline & & \multicolumn{2}{|c|}{ Internal primer in primer $1387 /$ RQ4CR product: RP1611 5'-CTTTGACCTGCTGCCAAGACT-3' } \\
\hline 55 & 433 & Q4C8 5'-GGAAATGTGCTGGGAAAGGAG-3' & Q4C5 5'-ACCAGTGCCTCAGGTGTCAGC-3' \\
\hline 55 & 989 & FP1387 5'-ATCTGTGTCTGCAGAGACGCC & 6R 5'-AGCAACTGGAGGCAGCTGTG-3' \\
\hline 61 & 310 & FP173T 5'-GATGCTGACACCTGAGGCAC-3 & RP1941 5'-GTGCCACGTCACTGGCAGTG-3' \\
\hline 55 & 797 & FP1871 5'-AGGTGAGCCATATGTGAACT-3' & 8R 5'-CTGCACGTAGCTCTCGAAGC-3' \\
\hline 55 & 873 & 7F 5'-CACAGCTGCCTCCAGTTGCT-3' & 8R 5'-CTGCACGTAGCTCTCGAAGC-3' \\
\hline 55 & 429 & 9F 5'-GCTTCGAGAGCTACGTGCAG-3' & 9R 5'-CTGTACGGTAAGCTGTATTG-3' \\
\hline 55 & 506 & E15FP 5'-AGAGCTGGTGTCCCCGTGGAC & Q4E15RP 5'-TGGGAACACGCGCTGTACCAG-3' \\
\hline 64 & 565 & FP2636 5'-CTCAAGAGGCTGAGCAGCTTAG & 10R 5'-GGCTCGTGGTCCCACTGCAG-3' \\
\hline 58 & 488 & 10F 5'-CAATACAGCTTACCGTACAG-3' & 10R 5'-GGCTCGTGGTCCCACTGCAG-3' \\
\hline 55 & 278 & FP2961 5'-TTTGACATGGTCAAGCTGGTGGA & RP3149 5'-CCATAGAGGAAGTCACATATCT-3' \\
\hline 61 & 492 & FP2961 5'-TTTGACATGGTCAAGCTGGTGGA & 11R 5'-AGGTCCTTGAGCCTGGTGCT-3' \\
\hline 55 & 423 & 11F 5'-CTGCAGTGGGACCACGAGCC-3' & 11R 5'-AGGTCCTTGAGCCTGGTGCT-3' \\
\hline 60 & 577 & 12F 5'-AGCACCAGGCTCAAGGACCT-3' & 12R 5'-GCCACCTGCAGGAGCTCTTC-3' \\
\hline 62 & 325 & FP3485 5'-CATCTTCCACGGCATCGG-3' & Q4A8RP-GTCACTGCCCTAGCCTCTGACAAC-3' \\
\hline
\end{tabular}

\section{Materials and methods}

Sequencing analysis was performed on DNA extracted from peripheral blood lymphocytes (Wizard $^{\circledR}$ Genomic, Promega). PCR was carried out with the primers reported in Table 1 based on the sequence (EMBL/GenBank accession number AB026546). The samples were then sequenced with the ABI PRISM ${ }^{\mathrm{TM}}$ BigDye Terminator Cycle Sequencing Ready Reaction Kit Ver 2 on ABI PRISM ${ }^{\mathrm{TM}} 310$ Genetic Analyser. Internal primers were used in the sequencing reactions.

$R T-P C R$ and sequencing: First strand cDNA was synthesised from $2 \mu \mathrm{g}$ of total RNA (Rnazolo-Gibco BRL Life Technologies Ltd., Paisley Scotland, UK) with oligodT primer (Riboclone cDNA Synthesis System, Promega, Madison, WI). Ten microliter of the first strand was amplified in a total volume of
$100 \mu \mathrm{l}$ containing specific mRNA RECQ4 primers (EMBL/GenBank accession number XM_005151.3|). Forward primer $5^{\prime}$-TGTCACAGTGAGGTCCCAGA is in exon 5 and the reverse primer $5^{\prime}$-ACACCTGGTCATCCATGAGT overlaps the junctions between exons 9 and 10 . Twenty amplification cycles were performed at an annealing temperature of $56^{\circ} \mathrm{C}$. The amplified $1044 \mathrm{bp}$ fragment was sequenced with the ABI PRISM $^{\mathrm{TM}}$ BigDye Cycle Sequencing Ready Reaction Kit Ver 2 on ABI PRISM ${ }^{\mathrm{TM}} 310$ Genetic Analyser.

\section{Case reports}

Rothmund-Thomson syndrome is a rare genodernatosis with variable clinical expression. The main features include: proportionate dwarfism, premature ageing, skin hyperpigmentation appearing within the first semester of life and longlife persisting, 
poikiloderma, telengiectasia, brittle hair sometimes progressing to total alopecia, distrophic nails, juvenile cataract, photosensitivity and congenital skeletal defects as hypoplasia or absence of the radii and thumbs.

This set of fraternal twins presented at the age of 10 months and based on the clinical features was diagnosed to have Rothmund-Thomson syndrome.

The male twin (IV-4) developed multicentric osteosarcoma at the age of 14 years involving the left humerus and the right tibia. He received chemotherapy and underwent resection of the limb salvage surgery for the tumour over the left humerus. However, he had to undergo an above knee amputation as the tibial tumour recurred. He is currently free of disease 5 years after stopping therapy.

His sister (IV-5) developed osteosarcoma of the left humerus at the age of 15 years. She also received chemotherapy and underwent limb salvage surgery. However, she developed metastases in her lungs and succumbed to the disease.

\section{Results}

The four generation pedigree with three members affected by in the last generation (Fig. 1) offered a unique chance to assess the link between RECQ4 gene and RTS. Cytogenetic analysis of the Malaysian kindred has been reported previously [4].

We amplified and sequenced the whole RECQ4 gene in the affected members of this family IV-4 and IV-5 (Fig. 1), and aligned the sequence with the reported genomic sequence [5]. We found two new mutations: a 2746-2756-delTGGGCTGAGGC in IVS8 (Fig. 2A), and a $5321 \mathrm{G} \rightarrow \mathrm{A}$ transition in exon 17 (Fig. 2B). The deletion shortens the IVS8 from 77 down to $66 \mathrm{bp}$. To evaluate the role of the intronic deletion in the splicing process we performed the RT-PCR analysis of RECQ4 gene: forward primer $5^{\prime}$-TGTCACAGTGAGGTCCCAGA is based on the sequence of exon 5 and the reverse primer 5'-ACACCTGGTCATCCATGAGT overlaps the junctions between exons 9 and 10. The amplified sequence spans from exons 5 to 9 . The spliced fragment is 1044 nts long (lane C), while the two affected members IV-4 and IV-5 share a fragment 1100 basepairs long. The father (lane III-1) has both the transcripts (Fig. 3). The sequence analysis shows that IVS8 has not been spliced out during transcription (Fig. 4). However, the deletion does not affect the canonical splicing elements, as the donor and the acceptor sites are conserved, as was the branch site (Fig. 4). As IVS8 is not spliced out, the deletion introduces a frameshift mutation and the translation terminates at a termination signal within the intron (Fig. 4), creating a truncated protein.

In the same family, we found another mutation a $5321 \mathrm{G} \rightarrow$ A transition leading to R1006Q missense mutation.

Analysis of the pattern of inheritance of the two mutations in the family members, whose samples was available for testing resulted in: II-2 and II-7, III-1 and III-2 and IV-1, IV-6, IV-8 being heterozygous, and IV-4 and IV-5 homozygous (Fig. 1). IV-7 is the only member tested in the last generation who did not inherited the RECQ4 deleted allele. The pattern of

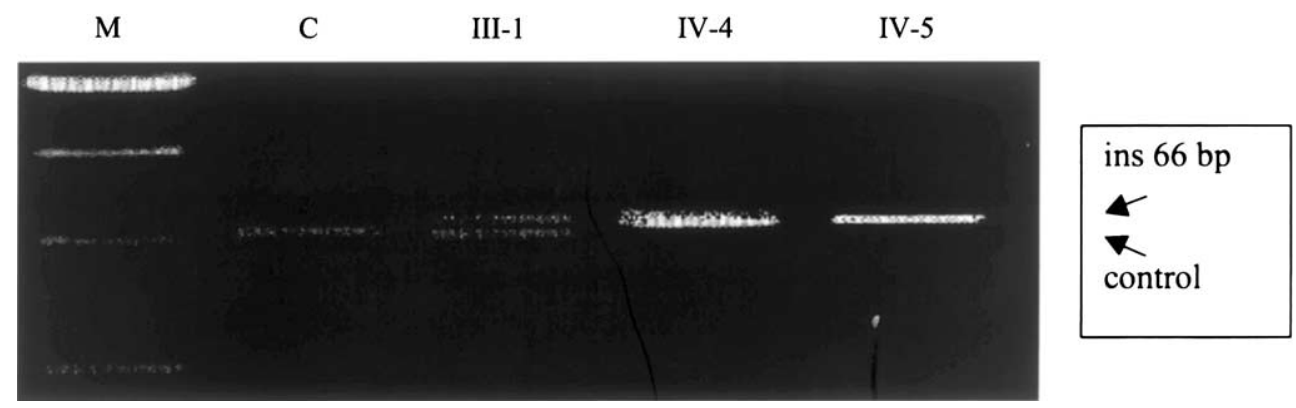

Fig. 3. Difference in electrophoretic mobility of the RT-PCR products spanning exons 5-9. Lane C: the correctly spliced fragment is 1044 bp long in the control. Lanes IV-4 and IV-5 show a 66 bp longer fragment due to the non-excision of IVS8. Lane III-1 shows two bands, according with the carrier status for the mutation (marker: M). 


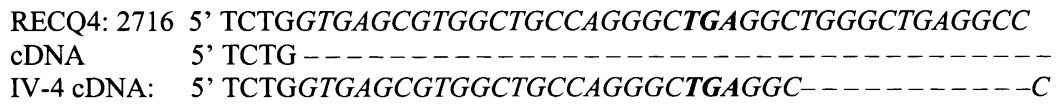

$\begin{array}{ll}\text { RECQ4: } 2759 & A G G C T G C A G A A C C C T G C T G C T G A C T C C C G C C C C A T C C A G G C A T \\ \text { cDNA } & \end{array}$

Fig. 4. Partial sequence of the RECQ4 human genomic sequence, including the donor and acceptor sites of IVS8. The RECQ4 human cDNA sequence following correct splicing and the cDNA sequence in patient IV-4. The intronic sequence is in italics and the TGA termination signal that terminates translation in the deleted alleles is in bold. Ten (GGGCTGAGGC) out of 11 (TGGGCTGAGGC) deleted residues are repeated in tandem.

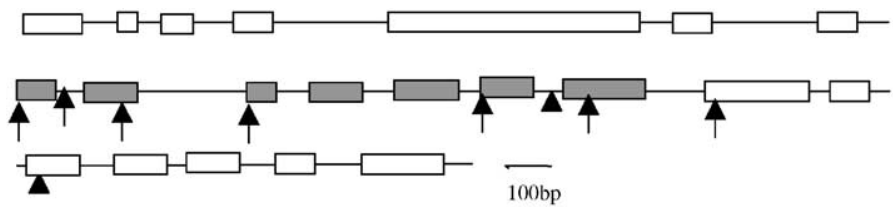

IVS7 Mut-6: Splicing mutation G-to-A transition, NT[2].

IVS8 Mut-7: Splicing mutation, 11-BP deletion NT 2746-2756 Current report.

Exon 9 Mut-5: Frameshift 1573T DEL [2].

Exon 10 Mut-1: Frameshift 7-BP DEL, NT 1650-1656) [1].

IVS12 Mut-4: Splicing mutation G-to-T transversion, NT4043 [1].

IVS12 Polymorphism-1: 30BP repeats, NT 3986-401 [1].

Exon 14 Mut-2: Non sense Q756TER, NT 2269 [1].

Exon 15 Mut-3: Frameshift 2-BP DEL, NT2492-3 [1].

Exon 17 Polymorphism-2: Missense R1006Q, NT 5321 Current report.

Fig. 5. Genomic structure of human RECQ4 gene. The boxes (1-21) and intervening lines indicate exons and introns. The total length is 6462 nucleotides (accession number AB026546). The filled boxes (8-14) indicate the helicase domain. The arrows indicate the mutations reported in RTS patients and the arrowheads the polymorphisms.

transmission is observed to be the same for both the mutations.

Fig. 5 presents a schematic diagram of the gene and a mutations update in RECQ4 gene. All mutations (except Mut-2) are in the helicase region of the gene spanning from exons 8 to $14[1,5]$.

\section{Discussion}

The gene responsible for RTS syndrome is RECQ4 (\#268400). The symbol (\#) before the entry number means that the phenotype can be caused by mutation in 2 or more genes. Mutations in RECQ4 gene are not associated with all patients with RTS except with the three families reported, thus, far to have compound heterozygous in their affected members, and the lack of mutations in some patients suggests a certain level of heterogeneity in the syndrome [3]: the present finding strengthens the association between this gene and RTS phenotype.

The pedigree (Fig. 1) shows a high degree of consanguinity: the parents of the affected are first cousins. This allowed us to trace back the transmission of the 
mutation in the family tree and to describe the first homozygous mutation in RECQ4 gene, present in two different affected individuals. In the same family studied, another child died of RTS, but no samples were available from this individual as he had passed away before the whole family was tested. Amplification and sequencing of RECQ4 gene has revealed the intronic $11 \mathrm{bp}$ deletion 2746-2756-delTGGGCTGAGGC (Fig. 2A). RT-PCR (Fig. 3) and sequencing results (Fig. 4) show that the IVS8 partial deletion affects splicing: RECQ4 mRNA results 66 residues longer and the reading frame is shifted introducing a termination signal (Fig. 4): the predicted product is 501 amino acids compared to the full-length 1208 amino acids [1]. Thus, the effect of this mutation would be "loss-of-function" type. This finding could account for the severe phenotype in this family: IV-2 died at age 7, IV-5 died at age 15 and the twin brother IV-4 at age 13 had a multicentric osteosarcoma. The other mutation running in the family is the $5321 \mathrm{G} \rightarrow \mathrm{A}$ transition in exon 17 leading to R1006Q missense mutation (Fig. 2B). This mutation is located downstream from the splice site mutation and would result in a premature stop codon in this family. This transition is considered a polymorphism as its presence has been identified in the normal population ( 3 out of 20 unrelated individuals tested). We also rule out any of its association with RTS as the mouse genome has a glutamine in the corresponding codon 1015 [6].

These considerations suggest that the IVS8 partial deletion is the only mutation responsible for the Rothmund-Thomson syndrome in this kindred.

RTPCR results show that the deletion leads to a wrong spliced mRNA and this would eventually lead to a truncated protein with very limited helicase domain. But how can an intronic deletion affect so severely the splicing machinery? The reported deletion is quite embedded within the intron (Fig. 4) 27 nts from the $5^{\prime}$ donor splice site and 40 nts from the $3^{\prime}$ acceptor splice site and does not alter the splice donor or the acceptor site or the branch site. Furthermore, it is almost duplicated inside the intron, 10 out of 11 bases are repeated in tandem (Fig. 4), and no variations have been found in the exon-regulatory sequences such as splicing enhancer elements [7].

Some new reports have associated a disease with an intronic mutation not affecting the canonical sites. In fact, the interest for these mutations has increased and may account for the some "heterogeneous" syndromes where intronic sequences could have been neglected. The very limited size of the intron, reduced from 77 to 66 bases, may account for the abnormal splicing [8], preventing the correct formation of the lariat. In a Thai family, a $20 \mathrm{bp}$ intronic deletion led to autosomal dominant polycystic kidney disease (ADPKD): the deletion prevents the transcription of the preceding exon 43 [9]. Another report showed that removal of GTAA residues from an intronic sequence in ATM gene caused a splicing processing defect; the deletion uncouples completely the U1 snRNP binding causing the activation of a cryptic exon [10]. These novel mutations describe how delicate the interactions between splicing and the intronic sequence are.

\section{Acknowledgements}

We thank Dr. Mak Joon Wah for having confidence in the project, Dr. Patricia Lim for fruitful discussions, Dr. Munirah Ahmad, Nitia Karunakaran, Harziah Ghazali, Tan Siew Kian, Siti Ruhana for their excellent assistance. We thank as well Dr. Paola Riva and Dr. Luisa Doneda for critically reading the manuscript.

\section{References}

[1] S. Kitao, A. Shimamoto, M. Goto, R.W. Miller, W.A. Smithson, N.M. Lindor, Y. Furuichi, Mutations in RECQL4 cause a subset of cases of Rothmund-Thomson syndrome, Nat. Genet. 22 (1999) 82-84.

[2] N.M. Lindor, Y. Furuichi, S. Kitao, A. Shimamoto, C. Arndt, S. Jalal, Rothmund-Thomson syndrome due to RECQ4 helicase mutations: report and clinical and molecular comparisons with Bloom syndrome and Werner syndrome, Am. J. Med. Genet. 90 (2000) 223-228.

[3] M.L. Wang, R.A. Levy, A. Gannavarapu, D.W. Stockton, L.M. Burks, D. Lev, C.M. Cunniff, S.E. Plon, Evidence for genetic heterogeneity in Rothmund-Thomson syndrome, Am. J. Med. Genet. 67 Suppl. (2000) A2107 376.

[4] M. Miozzo, P. Castorina, P. Riva, L. Dalpra, A.M. Fuhrman Conti, L. Volpi, A. Khoo, J. Wiegant, C. Rosenberg, L. Larizza, Chromosomal instability in fibroblasts and mesenchymal tumors from 2 sibs with Rothmund-Thomson syndrome, Int. J. Cancer 77 (1998) 504-510.

[5] S. Kitao, N.M. Lindor, M. Shiratori, Y. Furuichi, A. Shimamoto, Rothmund-Thomson syndrome responsible gene, RECQL4: genomic structure and products, Genomics 61 (1999) 268-276. 
[6] T. Ohhata, R. Araki, R. Fukumura, A. Kuroiwa, Y. Matsuda, M. Abe, Cloning genomic structure and chromosomal localization of the gene encoding mouse DNA helicase RECQL5beta, Gene 280 (2001) $59-66$.

[7] H.X. Liu, L. Cartegni, M.Q. Zhang, A.R. Krainer, A mechanism for exon skipping caused by nonsense or missense mutations in BRCA1 and other genes, Nat. Genet. 27 (2001) $55-58$.
[8] M. Deutsch, M. Long, Intron-exon structures of eukaryotic model organisms, Nucl. Acids Res. 27 (1999) 3219-3228.

[9] N. Rungroj, W. Thongnoppakhun, K. Vareesangthip, C. Sirinavin, P. Wilairat, P.T. Yenchitsomanus, Molecular defect of PKD1 gene resulting in abnormal RNA processing in a Thai family, J. Med. Assoc. Thai. 84 (2001) 1308-1316.

[10] F. Pagani, E. Buratti, C. Stuani, R. Bendix, T. Dörk, F.E. Baralle, A new type of mutation causes a splicing defect in ATM, Nat. Genet. 30 (2002) 426-429. 